\title{
Therapeutic efficacy of Bifidobacterium longum-mediated human interleukin-2 with endostatin or TRAIL in transplanted tumors in mice
}

\author{
YAN YIN $^{1}$, LEI KOU ${ }^{1}$, JIAN-JUN WANG ${ }^{1}$ and GEN-XING XU ${ }^{2,3}$ \\ ${ }^{1}$ Department of Biological Science and Technology and State Key Laboratory of Pharmaceutical Biotechnology, \\ School of Life Sciences; ${ }^{2}$ Center for Public Health Research, Medical School, and State Key Laboratory \\ of Analytical Chemistry for Life Science, Nanjing University, Nanjing 210093; \\ ${ }^{3}$ Jiangsu Research Center for Gene Pharmaceutical Engineering and Technology, Suzhou 215128, P.R. China
}

Received October 2, 2011; Accepted November 28, 2011

DOI: 10.3892/etm.2011.421

\begin{abstract}
Interleukin-2 (IL-2), as an important cytokine in immune response, has been demonstrated to have therapeutic activity in several cancer models. In our previous study, we showed that the pBV22210 vector containing a chloramphenicol resistance gene and the cryptic plasmid, pMB1, from the Bifidobacterium longum (B. longum) strain could stably replicate and did not significantly affect the biological characteristics of B. longum. In this study, B. longum was transfected by electroporation with pBV22210 containing IL-2 (B. longum-pBV22210-IL-2), its growth curve was determined, and its inhibitory effect on tumor xenografts in mice was examined. The results showed that B. longum-pBV22210-IL-2 reduced the tumor size and prolonged the survival time of $\mathrm{H} 22$ tumorbearing mice. In addition, when cyclophosphamide (CTX), $B$. longum-pBV22210-endostatin, or B. longum-pBV22210-TRAIL was combined with $B$. longum-pBV22210-IL-2, the antitumor effect was significantly enhanced. The survival times of the mice in the combination groups of B. longum-pBV22210-endostatin or B. longum-pBV22210-TRAIL were longer than those of the mice in the B. longum-pBV22210-IL-2 alone group. However, when CTX was added, the survival times of the mice showed no statistically significant difference compared with those of the mice in the dextrose-saline solution group. These results suggest that B. longum-pBV22210-IL-2 has potent antitumor effects that could be enhanced when combined with chemotherapeutic drugs or other antitumor genes.
\end{abstract}

Correspondence to: Professor Gen-Xing Xu, Center for Public Health Research, Medical School, Nanjing University, 22 Hankou Road, Nanjing 210093, P.R. China

E-mail: genxingxu@nju.edu.cn

Key words: Bifidobacterium longum, interleukin-2, endostatin, tumor necrosis factor-related apoptosis-inducing ligand, tumor, gene therapy, synergistic interactions

\section{Introduction}

Interleukin-2 (IL-2) was first identified in 1976 as a growthpromoting cytokine for bone marrow-derived Tlymphocytes (1), and the major function of IL-2 is to promote proliferation of $\mathrm{CD}^{+}$and $\mathrm{CD}^{+} \mathrm{T}$ cells (2). IL-2 also has functions on natural killer (NK) cells, B cells, lymphokine-activated killer (LAK) cells, monocytes and neutrophils (3-7). Accordingly, IL-2 plays a key role in the immune system, which has a close relationship with cancer. Although IL-2 has been used in the treatment of a number of different types of cancer, alone or in combination (8-10), it still has a number of limitations that restrict its full clinical use. Systemic injections, which are the most common routes for IL-2 used in cancer immunotherapy, have been reported to cause certain severe side-effects and toxicities including fever, nausea, capillary leak syndrome, diarrhea, chills, weight gain and hypotension $(11,12)$. To reduce the side-effects of IL-2, different strategies have been explored. However, the majority of these strategies have so far, been disappointing. Safer and more effective routes of IL-2 treatment are still required.

Hypoxic regions are characteristic of numerous types of solid tumors. As a non-pathogenic and anaerobic bacterium, Bifidobacterium longum (B. longum) is capable of selectively localizing and proliferating within the hypoxic regions of tumors $(13,14)$. However, the fact that exogenous plasmids cannot replicate stably in B. longum limits its application as a specific gene delivery system for cancer gene therapy. Though some shuttle vectors have been constructed over the years, only some of them could express foreign genes in B. longum successfully (15-18).

In our previous studies, we constructed the plasmids pBV22210-endostatin and pBV22210-tumor necrosis factor-related apoptosis-inducing ligand (TRAIL), which were proven to express stably when electroporated into B. longum. Furthermore, both B. longum-pBV22210-endostatin and B. longum-pBV22210-TRAIL showed a strong inhibitory effect on the growth of solid mouse tumors in vivo $(19,20)$. In this study, B. longum was transfected by electroporation with pBV22210 containing IL-2 (B. longum-pBV22210-IL-2), 
its growth curve was determined and its inhibitory effect on tumor xenografts in mice was examined. Furthermore, B. longum-pBV22210-endostatin, B. longum-pBV22210TRAIL or the chemotherapeutic drug cyclophosphamide (CTX) were combined with B. longum-pBV22210-IL-2 and their antitumor activity was also examined.

\section{Materials and methods}

Bacterial strains and plasmid. The Escherichia coli (E. coli) strain DH5 $\alpha$, wild-type (WT) B. longum, B. longumpBV22210-endostatin, B. longum-pBV22210-TRAIL and plasmid pcDNA3.0-IL-2 were maintained in our laboratory.

Reagents and enzymes. Primers were synthesized by Shanghai Shenergy Biocolor BioScience and Technology Company (Shanghai, China). CTX was purchased from Jiangsu Hengrui Medicine Co, Ltd. (Lianyungang, China). 2X Taqmix was purchased from Tiangen Biotech (Beijing, China), T4 DNA ligase and restriction endonucleases were purchased from Takara Bio (Dalian, China).

Animals and tumors. Male Kunming mice (20 $\pm 2 \mathrm{~g})$ were obtained from the Qinglongshan Animal Center (Nanjing, China). The animal experiments performed in this study were approved by the Nanjing University Animal Care and Use Committee. The animals were stored in an animal room that was maintained at a constant temperature with a $12 \mathrm{~h}$ light/ dark cycle and supplied with laboratory chow and water. Murine hepatoma cells (H22) were supplied by the Shanghai Academy of Medical Industry (Shanghai, China). The tumor model was established by subcutaneous injection of $\mathrm{H} 22$ tumor cells $\left(1 \times 10^{6}\right.$ cells $\left./ 0.2 \mathrm{ml}\right)$ into the right flank of each mouse.

\section{Construction of $p B V 22210-I L-2$}

PCR amplification of IL-2 gene. The plasmid pcDNA3.0-IL-2 was extracted following the instructions of the manufacturer and used as a template for PCR to amplify the IL-2 gene. The PCR primers and conditions were as follows: 5'-CCGGAATGT ACAGGATGCAACTCCTGTCTTG-3' (sense) and 5'-CGCG GATCCTTATCAAGTTAGTGTTGAGATGATG-3' (antisense), $94^{\circ} \mathrm{C}$ for $30 \mathrm{sec}, 53^{\circ} \mathrm{C}$ for $30 \mathrm{sec}$ and $72^{\circ} \mathrm{C}$ for $30 \mathrm{sec}$, for 30 cycles. PCR products were analyzed by electrophoresis on $1 \%$ agarose gels.

Ligation of $p B V 22210$ and $I L-2$. The PCR products and pBV22210-endostatin were digested by EcoRI/BamHI and then the gel-purified IL-2 and pBV22210 fragments were ligated at the EcoRI/BamHI site using T4 DNA ligase, yielding pBV22210-IL-2 (Fig. 1). The ligation solution was subsequently transferred into competent cells of DH5 $\alpha$, and pBV22210-IL-2 was detected on LB agar plates with $10 \mathrm{mg} / \mathrm{ml}$ chloramphenicol.

Identification of $p B V 22210-I L-2$. Following 12-14 h of incubation at $37^{\circ} \mathrm{C}$ under anaerobic conditions, positive clones of pBV22210-IL-2 with chloramphenicol resistance on LB agar were picked out and cultured overnight to extract the plasmids. The plasmid pBV22210-IL-2 was digested with EcoRI/BamHI and the digestion solution was analyzed by electrophoresis on $1 \%$ agarose gels. The constructed pBV22210-IL-2 plasmid was confirmed by sequencing.
Electroporation. Electrocompetent cells of B. longum were prepared according to the protocol of Rossi et al (21). Briefly, bacteria were resuspended in approximately $1 / 100$ of the original culture volume of ice-cold $0.5 \mathrm{M}$ sucrose plus $1 \mathrm{mM}$ ammonium citrate ( $\mathrm{pH}$ 6.0). The $\mathrm{pBV} 22210-\mathrm{IL}-2$ plasmids were added to the bacteria suspension and incubated at $4^{\circ} \mathrm{C}$ for $2-3 \mathrm{~h}$. The mixture was stored in a pre-cooled sterile Gene Pulser disposable cuvette (interelectrode distance $0.2 \mathrm{~cm}$; Bio-Rad, Hercules, CA, USA). The pBV22210-IL-2 plasmids were transferred directly into $B$. longum by electroporation in a Bio-Rad Gene-Pulser apparatus at $25 \mu \mathrm{F}$ and $2.5 \mathrm{kV}$ with the pulse controller set at $200 \Omega$. Subsequently, the mixture was inoculated in trypticase-peptoneyeast (TPY) culture and anaerobically cultivated overnight at $37^{\circ} \mathrm{C}$. Diluted overnight culture was plated on TPY agar plates and monoclones were cultivated alternately in TPY culture with and without $5 \mu \mathrm{g} / \mathrm{ml}$ chloramphenicol for at least 10 generations. Then stable B. longum-pBV22210-IL-2 with chloramphenicol resistance could be obtained.

Growth assay of B. longum- $p B V 22210-I L-2$. Overnight culture of B. longum-pBV22210-IL-2 in TPY medium was inoculated into fresh medium with or without $5 \mu \mathrm{g} / \mathrm{ml}$ chloramphenicol to an initial optical density (OD) value of 0.010 at $600 \mathrm{~nm}$ (OD 600). The cultures were grown under anaerobic conditions at $37^{\circ} \mathrm{C}$ for $20 \mathrm{~h}$ and $\mathrm{OD}$ values were measured every hour. WT B. longum cells were used as the control.

Antitumor activity of B. longum-pBV22210-IL-2. Mice were weighed and randomly divided into seven experimental groups (six per group) following hypodermic inoculation of H22 cells for $24 \mathrm{~h}$. One negative control group was injected with dextrose-saline solution ( $0.4 \mathrm{ml} /$ day, i.v., on days $1-10)$, and six treated groups were injected with WT B. longum $(0.4 \mathrm{ml} / \mathrm{day}$, i.v., on days 1-10), B. longum-pBV22210-IL-2 (0.4 ml/day, i.v, on days 1-10), CTX (30 mg/kg, i.p., on days 1, 3, 5, 7 and 9), B. longum-pBV22210-IL-2 (0.4 ml/day, i.v., on days 1,3 , 5, 7 and 9) plus B. longum-pBV22210-TRAIL (0.4 ml/day, i.v., on days 2, 4, 6, 8 and 10), B. longum-pBV22210-IL-2 (0.4 ml/day, i.v, on days $1,3,5,7$ and 9) plus B. longumpBV22210-endostatin (0.4 ml/day, i.v., on days 2, 4, 6, 8 and 10), and $B$. longum-pBV22210-IL-2 (0.4 ml/day, i.v., on days 1,3, 5, 7 and 9) plus CTX (30 mg/kg, i.p., on days 1, 3, 5, 7 and 9), respectively. All B. longum cells were washed and resuspended with dextrose-saline solution at a concentration of $2.5 \times 10^{8}$ cells $/ \mathrm{ml}$ prior to injection. CTX was suspended with dextrose-saline solution and $0.2 \mathrm{ml}$ of the solution was injected each time per mouse. The mice were sacrificed $72 \mathrm{~h}$ following the last administration. The tumors were excised and weighed. The level of inhibition of tumor growth was determined by the following formula:

tumor weight of control group - tumor weight of treatment group tumor weight of control group

Effect of B. longum-pBV22210-IL-2 on the survival time of H22 tumor-bearing mice. Mice were randomly divided into seven experimental groups (10 per group) and mice in each group were injected respectively, as mentioned above. The mice were kept after the last administration and their survival time was recorded until day 70. All mice were sacrificed on day 71 and the survival data were analyzed. 


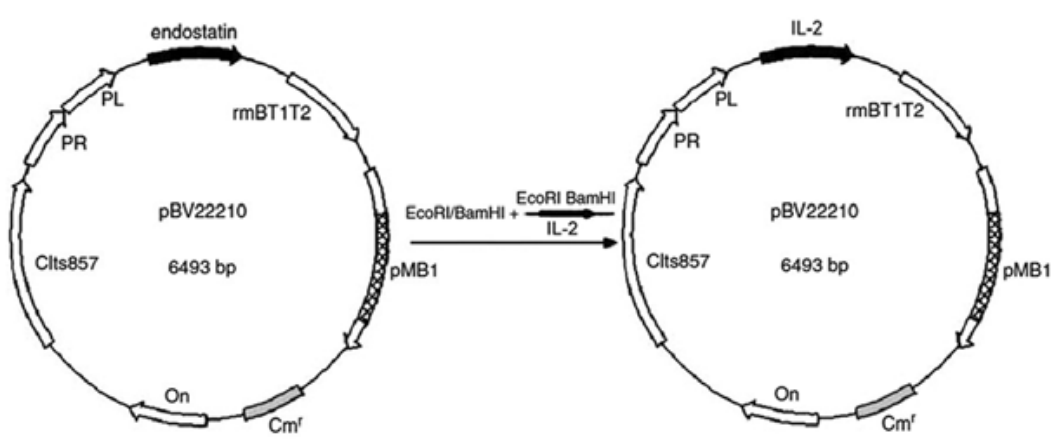

Figure 1. Construction of the expression vector, pBV22210-IL-2.

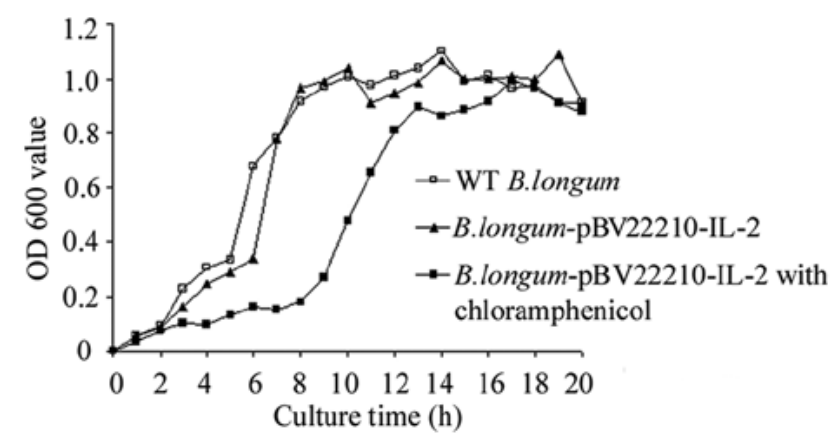

Figure 2. The growth curves of B. longum-pBV22210-IL-2 and wild-type (WT) B. longum cells. B. longum-pBV22210-IL-2 cells were incubated anaerobically at $37^{\circ} \mathrm{C}$ in trypticase-peptone-yeast (TPY) medium with or without chloramphenicol. WT B. longum cells were incubated anaerobically at $37^{\circ} \mathrm{C}$ in TPY medium without selective pressure. OD 600, optical density at $600 \mathrm{~nm}$.

Statistical analysis. The results were expressed as the means \pm SD value. The data were statistically analyzed using the Student's t-test in both groups and the ANOVA test in multiple groups. The comparisons among multiple groups were performed using Student-Newman-Keuls q-test. Survival analysis was performed using SPSS 15.0. $\mathrm{P}<0.05$ was considered to indicate a statistically significant difference.

\section{Results}

Identification of the pBV22210-IL-2 plasmid. The constructed pBV22210-IL-2 plasmid (Fig. 1) was confirmed by sequencing, and the sequencing result was as follows: GAATTCCAAA TGTACAGGATGCAACTCCTGTCTTGCATTGCACTAAG TCTTGCACTTGTCACAAACAGTGCACCTACTTCAAGT TCTACAAAGAAAACACAGCTACAACTGGAGCATTTA CTGCTGGATTTACAGATGATTTTGAATGGAATTAATA ATTACAAGAATCCCAAACTCACCAGGATGCTCACATT TAAGTTTTACATGCCCAAGAAGGCCACAGAACTGAA ACATCTTCAGTGTCTAGAAGAAGAACTCAAACCTCT GGAGGAAGTGCTAAATTTAGCTCAAAGCAAAAACT TTCACTTAAGACCCAGGGACTTAATCAGCAATATCAA CGTAATAGTTCTGGAACTAAAGGGATCTGAAACAAC ATTCATGTGTGAATATGCTGATGAGACAGCAACCATT GTAGAATTTCTGAACAGATGGATTACCTTTTGTCAAA GCATCATCTCAACACTAACTTGATAAGGATCC. Restriction site: EcoRI GAATTC; BamHI GGATCC.
The sequencing result showed that the recombinant plasmid pBV22210-IL-2 was successfully constructed. The sequence of the IL-2 gene between the EcoRI and BamHI sites was consistent with the GenBank report.

Growth characteristics. The growth curves of B. longumpBV22210-IL-2 and WT B. longum were similar in the TPY medium without selective pressure, whereas the lag phase of B. longum-pBV22210-IL-2 in the selective medium was statistically longer than that in the non-selective medium as shown in Fig. 2. Both B. longum-pBV22210-IL-2 and WT B. longum in the TPY medium without chloramphenicol grew to an exponential phase after $3 \mathrm{~h}$ and a stationary phase (OD 600=1.1) after 8 h of incubation. However, B. longum-pBV22210-IL-2 cells in the TPY medium with chloramphenicol $(5 \mu \mathrm{g} / \mathrm{ml})$ grew to an exponential phase after $7 \mathrm{~h}$ and a stationary phase after $11 \mathrm{~h}$ of incubation.

Effect of B. longum-pBV22210-IL-2 on growth of H22 tumors. We established an H22 tumor xenograft model to assess the antitumor efficacy of B. longum-pBV22210-IL-2 alone and in combination with CTX, B. longum-pBV22210-endostatin or B. longum-pBV22210-TRAIL in vivo. The tumors excised from each group are shown in Fig. 3A. The average tumor weight of each group is shown in Fig. 3B. Compared with the dextrose-saline solution group, the tumor inhibition rate of the B. longum-pBV22210-IL-2 group was $56.2 \%(\mathrm{p}<0.05)$, which was higher than that of the WT B. longum group $(32 \%)(\mathrm{p}>0.05)$, but lower than that of the CTX group $(62.2 \%)(\mathrm{p}<0.05)$. Compared with the dextrose-saline solution group, the inhibitory rate of the co-injection of B. longum-pBV22210-IL-2 with B. longum-pBV22210-TRAIL reached $74 \%(\mathrm{p}<0.05)$, while that of B. longum-pBV22210-IL-2 with B. longumpBV22210-endostatin was $77.5 \%(\mathrm{p}<0.05)$ and that of B. longum-pBV22210-IL-2 with CTX was $82.7 \%(\mathrm{p}<0.05)$. Compared with the B. longum-pBV22210-IL-2 alone group, the tumor inhibition rate of the B. longum-pBV22210-IL-2 plus B. longum-pBV22210-TRAIL group was $17.8 \%(\mathrm{p}<0.05)$ higher, whereas that of the B. longum-pBV22210-IL-2 plus B. longum-pBV22210-endostatin group was $21.3 \%(\mathrm{p}<0.05)$ higher and the $B$. longum-pBV22210-IL-2 plus CTX group was $26.5 \%(\mathrm{p}<0.05)$ higher. These results suggest that B. longum-pBV22210-IL-2 alone can inhibit tumor growth significantly, and when combined with other drugs, the inhibitory efficacy is even greater. 


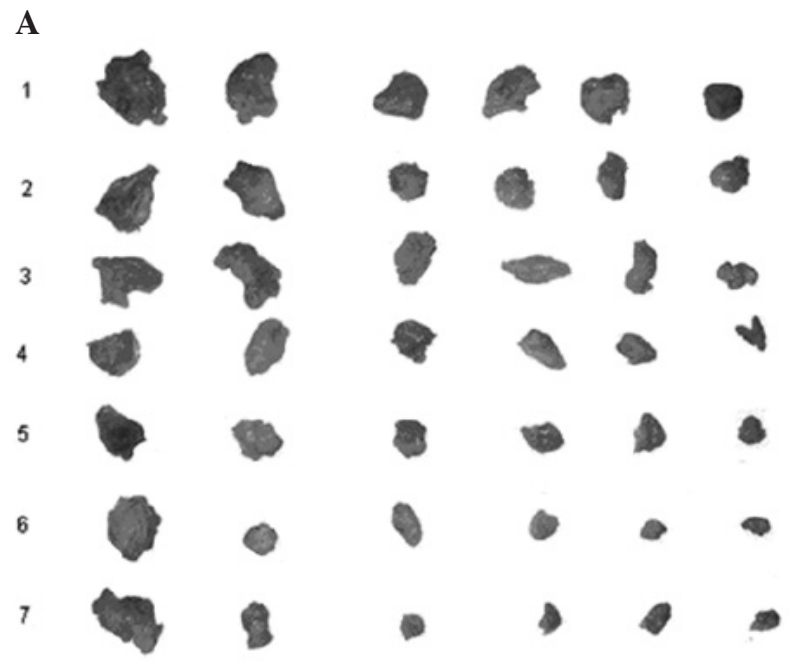

B

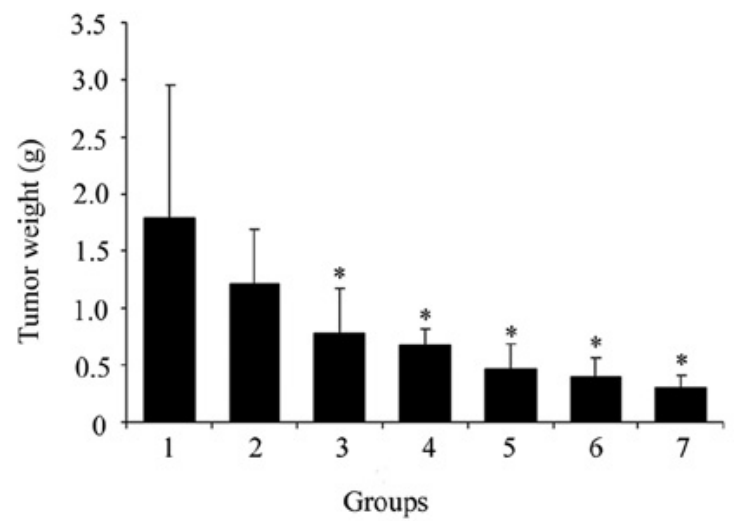

Figure 3. The suppression effects of B. longum-pBV22210-IL-2 on H22 cell tumor growth. There were six mice in each group. The tumor weight was measured for each mouse. (A) Tumors excised from different groups. (B) The average tumor weight of different groups. Numbers 1-7 in the $\mathrm{X}$ axis correspond to the dextrose-saline solution group, wild-type B. longum group, B. longum-pBV22210-IL-2 group, CTX (30 mg/kg) group, B. longum-pBV22210-IL-2 plus B. longum-pBV22210-TRAIL group, B. longum-pBV22210-IL-2 plus B. longum-pBV22210-endostatin group and B. longum-pBV22210-IL-2 plus CTX $(30 \mathrm{mg} / \mathrm{kg}$ ) group, respectively. "P<0.05 versus dextrose-saline solution group. IL-2, interleukin-2; CTX, cyclophosphamide.

Effect of B. longum-pBV22210-IL-2 on survival time of $H 22$ tumor-bearing mice. To examine the effect of B. longumpBV22210-IL-2 on the survival time of tumor-bearing mice, we developed a xenograft model in which $\mathrm{H} 22$ cells were injected subcutaneously into Kunming mice. The survival curves of four single administration groups are shown in Fig. 4A. The mean survival time of the dextrose-saline solution group was $20.4 \pm 1.2$ days, and that of the CTX group extended to $23.1 \pm 1.6$ days, but the difference was not statistically significant ( $>0.05$ ). The mean survival time of the WT B. longum and $B$. longum-pBV22210-IL-2 groups extended to $26.8 \pm 2.1$ $(\mathrm{p}<0.01)$ and $31.8 \pm 3.1$ days $(\mathrm{p}<0.01)$, respectively. The survival curves of the co-administration groups are shown in Fig. 4B. The mean survival time of the B. longum-pBV22210-IL-2 plus CTX group, the $B$. longum-pBV22210-IL-2 plus B. longumpBV22210-endostatin group and the $B$. longum-pBV22210IL-2 plus B. longum-pBV22210-TRAIL group was $28.4 \pm 2.4$, $34.7 \pm 3.6$ and $35.6 \pm 3.4$ days, respectively. The H22 tumorbearing mice co-administered $B$. longum-pBV22210-IL-2 with B. longum-pBV22210-endostatin or B. longum- pBV22210TRAIL had significantly improved survival compared with the mice administered dextrose-saline solution $(\mathrm{p}<0.05)$. However, the survival time of the mice in the B. longumpBV22210-IL-2 plus CTX group was not statistically significant compared with the mice in the dextrose-saline solution group (p>0.05). Compared with the WT B. longum group, the B. longum-pBV22210-IL-2 plus B. longum-pBV22210endostatin group and the $B$. longum-pBV22210-IL-2 plus B. longum-pBV22210-TRAIL group showed statistical significance $(\mathrm{p}<0.05)$, while the $B$. longum-IL-2 plus CTX group showed no statistical significance $(\mathrm{p}>0.05)$.

\section{Discussion}

The immune system plays a major role in tumorigenesis and metastasis. As a cytokine closely related to the immune system, IL-2 has been used in the treatment of a number of different
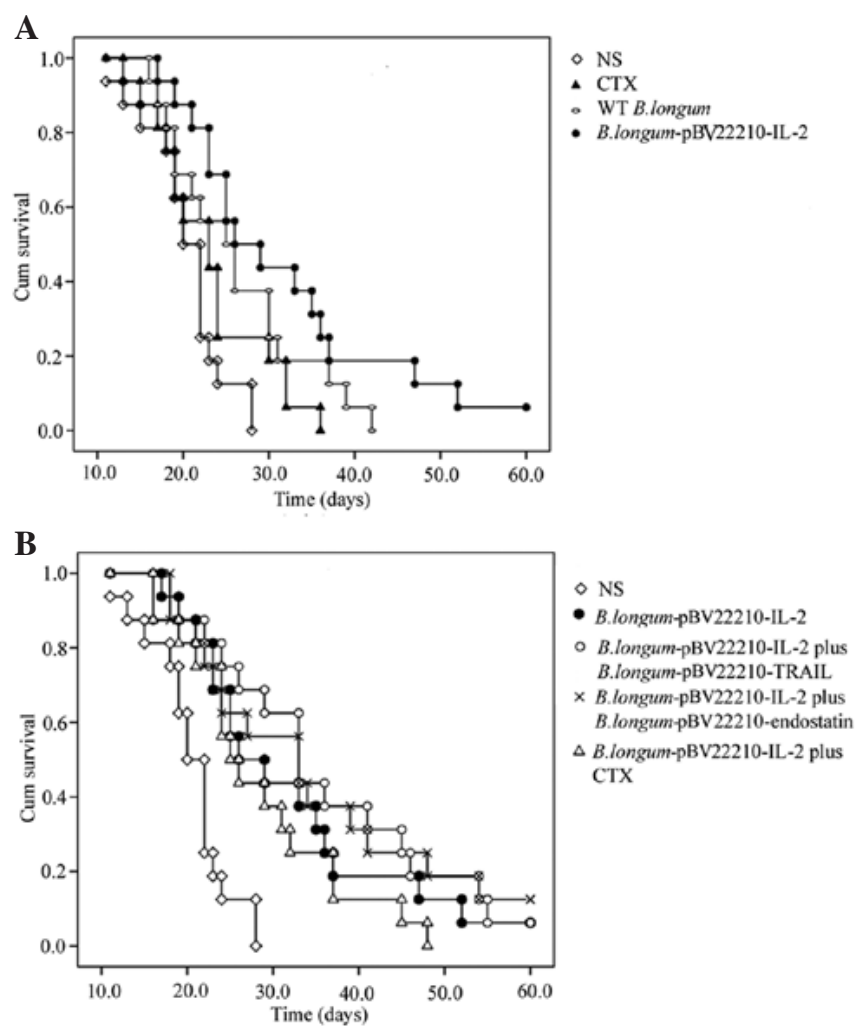

Figure 4. Kaplan-Meier survival curves of H22 tumor-bearing mice. (A) A Kaplan-Meier survival curve of mice treated with dextrose-saline solution, wild-type B. longum, B. longum-pBV22210-IL-2 and CTX $(30 \mathrm{mg} / \mathrm{kg})$, respectively. (B) A Kaplan-Meier survival curve of mice treated with dextrose-saline solution, $B$. longum-pBV22210-IL-2, B. longum-pBV22210-IL-2 plus $B$. longum-pBV22210-TRAIL, B. longum-pBV22210-IL2 plus B. longum-pBV22210-endostasin and B. longum-pBV22210-IL2 plus CTX (30 mg/kg), respectively. IL-2, interleukin-2; CTX, cyclophosphamide.

types of cancer, including metastatic melanoma (8), mesothelioma (9), lymphoma (10), kidney carcinoma (22), hepatocellular carcinoma (23), lung cancer (24) and bladder carcinoma (25). 
However, toxicity and side-effects remain the major drawbacks of IL-2 therapy. To maximize the therapeutic efficacy and minimize the systemic side-effects of IL-2, several delivery systems have been used. These delivery systems have included gene therapy, and liposome and polymeric microspheres (26-28), among which safety and efficacy remain a problem.

As an important non-pathogenic anaerobic bacterium in the human colon, Bifidobacterium has been used as a delivery system for a number of functional genes in cancer gene therapy (29). In a previous study, Li et al (30) used Bifidobacterium adolescentis (B. adolescentis) as the delivery system to transport the endostatin gene to solid tumors. When $B$. adolescentis with the endostatin gene was injected into tumor-bearing mice through the tail vein, they were only found in the tumors and no bacilli were found in other normal tissues. These results further prove that Bifidobacterium only germinates and proliferates in solid tumors and might be a highly specific and efficient vector for anticancer genes in cancer gene therapy. Furthermore, Bifidobacterium itself has been proven to have anticancer functions (31). Bifidobacterium has anticancer effects as not only has it been shown to inhibit cancer growth directly but it also to increase the activity of a number of immune cells and factors including IL-2 (32). In view of the properties above, B. longum may be used as a nontoxic, efficient and targeted delivery system of IL-2.

A main obstacle in the application of B. longum as a gene delivery system is the instability of exogenous plasmids and the low level expression of the exogenous gene. A number of attempts have been made to solve this problem. Nakamura et al (33) constructed the plasmid, pBLES100-S-eCD, which included the histone-like protein (HU) gene promoter and the gene encoding the cytosine deaminase (CD) of E. coli in the pBLES100 vector. Using this vector, the expression of cytosine deaminase in B. longum successfully converted 5-fluorocytosine (5-FC) to 5-fluorouracil (5-FU). Yi et al (34) successfully constructed a Bifidobacterium infantis/CD targeting gene therapy system with a recombinant $\mathrm{CD} / \mathrm{pGEX}-1 \lambda \mathrm{T}$ plasmid, which was used in the inhibition of melanoma in vitro and in vivo. In our laboratory, $\mathrm{Xu}$ et al (35) constructed a pBV22210-endostatin vector combining a chloramphenicol resistance gene $\left(\mathrm{Cm}^{\mathrm{r}}\right)$ from a pBCSK $(\mathrm{t})$ plasmid and a cryptic plasmid pMB1 from the WT B. longum strain. When electroporated into $B$. longum, the plasmid could be stably expressed and B. longum-pBV22210-endostatin exhibited a strong inhibitory effect on H22 tumor growth. Hu et al (20) constructed a B. longum strain with pBV22210 encoding the extracellular domain of TRAIL (B. longum-pBV22210-TRAIL), and it selectively proliferated in the tumor tissue and exhibited a definite antitumor effect on S180 osteosarcoma. In this study, we constructed the plasmid, pBV22210-IL-2, based on the plasmid, pBV22210-endostatin. The results showed that the growth curves of B. longum-pBV22210-IL-2 and WT B. longum were similar, which suggested that the biological characteristics of $B$. longum were not affected after the plasmid was electroporated. The result that B. longum-pBV22210-IL-2 could grow in TPY medium with chloramphenicol for at least 10 generations further proved that pBV22210-IL-2 was constructed successfully and could be stably expressed in B. longum.

The antitumor effect of B. longum-pBV22210-IL-2 was evaluated in $\mathrm{H} 22$ tumor-bearing mice in vivo. B. longumpBV22210-IL-2 inhibited tumor growth by $56.2 \%(\mathrm{p}<0.05)$, and extended the mean survival time of tumor-bearing mice to $31.8 \pm 3.1$ days $(\mathrm{p}<0.01)$. These results suggest that B. longum-pBV22210-IL-2 has a significant effect on H22 tumor cells. As B. longum-pBV22210-TRAIL and B. longum-pBV22210-endostatin were also proven to have an antitumor effect in our previous studies, we assessed the synergistic effect of a combination of B. longumpBV22210-IL-2 and B. longum-pBV22210-TRAIL or B. longum-pBV22210-endostatin on H22 tumors. IL-2, TRAIL and endostatin are tumor suppressor genes with different mechanisms. IL-2 activates immune cells and regulated the body's immune function, TRAIL activates related apoptotic pathways of tumor cells and induces apoptosis, and endostatin inhibits angiogenesis and reduces nutrient supply to the tumor. Our results showed that the tumor growth inhibition was enhanced and survival time was prolonged in the combination treatment groups, which further proved that co-administration of drugs with different mechanisms could be a feasible method in cancer therapy. In addition, as chemotherapy remains the major approach to treat tumors in clinical practice, we used the chemotherapeutic drug, CTX, combined with B. longum-pBV22210-IL-2 and assessed their synergistic effect on $\mathrm{H} 22$ tumors. The results showed that the combination of CTX and B. longum-pBV22210-IL-2 significantly inhibited tumor growth; however, the survival time of the tumor-bearing mice administered this combination was not extended. In our previous studies, we combined CTX with B. longum-pBV22210-granulocyte colony-stimulating factor (GCSF) to observe the effects on H22 and S180 tumor-bearing mice, and similar results were obtained (36). These results were attributed to the toxicity of CTX. Lower dose and a combination of targeted gene therapy may solve the severe side-effects of chemotherapeutic drugs.

In conclusion, we established a new delivery system of IL-2 by B. longum and developed a strategy of combining IL-2 with other antitumor genes or chemotherapeutic drugs for tumor treatment. Treatment with B. longum-pBV22210-IL-2 resulted in significant inhibition of tumor growth and longer survival time in tumor-bearing mice. The combination treatments had much greater antitumor effects. Our results support the potential of B. longum as a gene delivery system and the combination of chemotherapy and target gene therapy for advancing the treatment of cancer.

\section{Acknowledgements}

This study was supported by a grant (BK2009109, SBK201123046) from the Natural Science Fund of Jiangsu Province to G.-X. X. and Y. Y.

\section{References}

1. Morgan DA, Ruscetti FW and Gallo R: Selective in vitro growth of T lymphocytes from normal human bone marrows. Science 193: 1007-1008, 1976.

2. Gaffen SL and Liu KD: Overview of interleukin-2 function, production and clinical applications. Cytokine 28: 109-123, 2004.

3. Robertson MJ and Ritz J. Biology and clinical relevance of human natural killer cells. Blood 76: 2421-2438, 1990.

4. Mingari MC, Gerosa F, Carra G, et al: Human interleukin-2 promotes proliferation of activated $\mathrm{B}$ cells via surface receptors similar to those of activated T cells. Nature 312: 641-643, 1984. 
5. Grimm EA, Mazumder A, Zhang HZ and Rosenberg SA Lymphokine-activated killer cell phenomenon-Lysis of natural killer-resistant fresh solid tumor cells by interleukin 2-activated autologous human peripheral blood lymphocytes. J Exp Med 155: 1823-1841, 1982.

6. Espinoza-Delgado I, Bosco MC, Musso T, Gusella GL, Longo DL and Varesio L: Interleukin-2 and human monocyte activation. J Leukoc Biol 57: 13-19, 1995.

7. Ferrante A: Activation of neutrophils by interleukins-1 and -2 and tumor necrosis factors. Immunol Ser 57: 417-436, 1992.

8. Radny P, Caroli UM, Bauer J, et al: Phase II trial of intralesional therapy with interleukin-2 in soft tissue melanoma metastases Brit J Cancer 89: 1620-1626, 2003.

9. Astoul P, Picat-Joossen D, Viallat JR and Boutin C: Intrapleura administration of interleukin-2 for the treatment of patients with malignant pleural mesothelioma: A Phase II study. Cancer 83: 2099-2104, 1998.

10. Gisselbrecht C, Maraninchi D, Pico JL, et al: Interleukin-2 treatment in lymphoma: A phase II multicenter study. Blood 83 2081-2085, 1994.

11. Rosenberg SA, Lotze MT, Muul LM, et al: A progress report on the treatment of 157 patients with advanced cancer using lymphokine-activated killer cells and interleukin-2 or high-dose interleukin-2 alone. N Engl J Med 316: 889-897, 1987.

12. Lotze MT, Matory YL, Rayner AA, et al: Clinical effects and toxicity of interleukin-2 in patients with cancer. Cancer 58: 2764-2772, 1986.

13. Kimura NT, Taniguchi S, Aoki K and Baba T: Selective localization and growth of Bifidobacterium bifidum in mouse tumors following intravenous administration. Cancer Res 40: 2061-2068, 1980.

14. Yazawa K, Fujimori M, Amano J, Kano Y and Taniguchi S: Bifidobacterium longum as a delivery system for cancer gene therapy: selective location and growth in hypoxic tumors. Cancer Gene Ther 7: 269-274, 2000.

15. Rossi M, Brigidi P, Rodriguez AGVY and Matteuzzi D Characterization of the plasmid pMB1 from Bifidobacterium longum and its use for shuttle vector construction. Res Microbiol 147: 133-143, 1996.

16. Rossi M, Brigidi P and Matteuzzi D: Improved cloning vectors for Bifidobacterium spp. Lett Appl Microbiol 26: 101-104, 1998.

17. Lee JH and O'Sullivan DJ: Sequence analysis of two cryptic plasmids from Bifidobacterium longum DJO10A and construction of a shuttle cloning vector. Appl Eeviron Microbiol 72: 527-535, 2006.

18. Matsumura H, Takeuchi A and Kano Y: Construction of Escherichia coli Bifidobacterium longum shuttle vector transforming B-longum 105-A and 108-A. Biosci Biotech Biochem 61 $1211-1212,1997$

19. Fu GF, Li X, Hou YY, Fan YR, Liu WH and Xu GX: Bifidobacterium longum as an oral delivery system of endostatin for gene therapy on solid liver cancer. Cancer Gene Ther 12 : 133-140, 2005.

20. Hu B, Kou L, Li C, et al: Bifidobacterium longum as a delivery system of TRAIL and endostatin cooperates with chemotherapeutic drugs to inhibit hypoxic tumor growth. Cancer Gene Ther 16: 655-663, 2009 .
21. Rossi M, Brigidi P and Matteuzzi D: An efficient transformation system for Bifidobacterium spp. Lett Appl Microbiol 24: 33-36, 1997.

22. Sosman JA, Kohler PC, Hank J, et al: Repetitive weekly cycles of recombinant human interleukin 2: Responses of renal carcinoma with acceptable toxicity. J Nat Cancer Inst 80: 60-63, 1988

23. Shirai M, Watanabe S and Nishioka M: Antitumour effect of intratumoral injection of human recombinant interleukin-2 in patients with hepatocellular carcinoma: A preliminary report. Eur J Cancer 26: 1045-1048, 1990.

24. Yasumoto K, Mivazaki K, Nagashima A, et al: Induction of lymphokine-activated killer cells by intrapleural instillations of recombinant interleukin-2 in patients with malignant pleurisy due to lung cancer. Cancer Res 47: 2184-2187, 1987.

25. Ferlazzo G, Magno C, Lupo G, et al: A phase I study of intravesical continuous perfusion of recombinant interleukin-2 in patients with superficial bladder cancer. Am J Clin Oncol 18: 100-104, 1995.

26. Hoffman DMJ and Figlin RA: Intratumoral interleukin 2 for renal-cell carcinoma by direct gene transfer of a plasmid DNA/ DMRIE/DOPE lipid complex. World J Urol 18: 152-156, 2000.

27. Johnston D, Reynolds SR and Bystryn JC: Interleukin-2/ liposomes potentiate immune responses to a soluble protein cancer vaccine in mice. Cancer Immunol Immunother 55: 412-419, 2006.

28. Hsu W, Lesniak MS, Tyler B and Brem H: Local delivery of interleukin-2 and adriamycin is synergistic in the treatment of experimental malignant glioma. J Neurooncol 74: 135-140, 2005.

29. Fu GF, Yin Y, Hu B and Xu GX: Bifidobacterium as a delivery system of functional genes for cancer gene therapy. In: Emerging Cancer Therapy: Microbial Approaches and Biotechnological Tools. Fialho A and Chakrabarty A (eds). John Wiley \& Sons, Inc., Hoboken, NJ, pp99-117, 2010.

30. Li X, Fu GF, Fan YR, et al: Bifidobacterium adolescentis as a delivery system of endostatin for cancer gene therapy: selective inhibitor of angiogenesis and hypoxic tumor growth. Cancer Gene Ther 10: 105-111, 2003.

31. Kohwi Y, Imai K, Tamura Z and Hashimoto Y: Antitumor effect of Bifidobacterium infantis in mice. Gann 69: 613-618, 1978.

32. Lopez P, Gueimonde M, Margolles A and Suárez A: Distinct Bifidobacterium strains drive different immune responses in vitro. Int J Food Microbiol 138: 157-165, 2010.

33. Nakamura T, Sasaki T., Fujimori M, et al: Cloned cytosine deaminase gene expression of Bifidobacterium longum and application to enzyme/pro-drug therapy of hypoxic solid tumors. Biosci Biotechnol Biochem 66: 2362-2366, 2002.

34. Yi C, Huang Y, Guo Z and Wang S: Construction of Bifidobacterium infantis/CD targeting gene therapy system. Chin-Germ J Clin Oncol 4: 244-247, 2005.

35. Xu YF, Zhu LP, Hu B, et al: A new expression plasmid in Bifidobacterium longum as a delivery system of endostatin for cancer gene therapy. Cancer Gene Ther 14: 151-157, 2007.

36. ZhuLP,Yin Y,Xing J, et al: Therapeutic efficacy of Bifidobacterium longum-mediated human granulocyte colony-stimulating factor and/or endostatin combined with cyclophosphamide in mousetransplanted tumors. Cancer Sci 100: 1986-1990, 2009. 